\title{
Adjustment of Animal Growth Rate Responses in Repeat Measurement Grazing Trials
}

\author{
David Scott
}

AgResearch, P.O. Box 60, Lincoln, New Zealand

\begin{abstract}
In animal-response grazing trials there are sources of uncertainty in one-period one-off measurements, which as partial factorisation over time, become traceable and quantifiable sources of variation in repeat measurement trials. This is illustrated for a trial comparing sheep and goat live-weight gains under two stocking rates on mixed species pastures established by three contrasting sowing methods. It used variable plot size to give uniform animal numbers and tracked changes in individual animal performance and pasture growth in different periods. It was repeated on the same plots and animals over 17 grazing cycles. The variation explainable was greater with growth rates expressed as percent live-weight increase per day, than as weight or metabolic weight increase per day. The base data sets were adjusted for specific weighing-day effects of estimated gut-fill using moving averages, and for calibration for individual animal effects by genotype/environment analysis. Collectively these significantly increased the proportion explainable by $3.1-3.8 \%$ in variance analyses using qualitative treatment variables, and $2.7-3.7 \%$ in response function analyses relative to measured climate, pasture, plot and collective animal covariates. Simulation studies, to allow for variability in the independent variables as well as the dependent variables, indicated that the proportion explainable could increase by a further $0-1.2 \%$ and $1.1-1.9 \%$ respectively for the variance or response function approaches.
\end{abstract}

Keywords: Experimental design, grazing trials, sheep, goats.

\section{INTRODUCTION}

There is continuing need to obtain animal performance data in evaluating new techniques, pasture mixtures, species or plant breeding material for their adaptation to particular environments or management conditions. A number of approaches or techniques have been developed.

The size of pasture trial areas required to carry adequate animal numbers for growth rate measurements can be a problem because of the resources required. The usual reasons are the need for sufficient number of animals and sufficient magnitude of weight changes to cover variability related to other factors e.g. between animal variability, effect of prevailing conditions on stomach fill, water balance, wool or fibre moisture content, instrument variability, observer variability, random effects, etc. These may be true in a oneoff one-period measurement. However, it is also generally true, both because of the high initial set-up costs and to determine sustainability effects that measurements repeated over time are also a feature of animal/pasture evaluation studies. It is usually the negative effects of confounding with treatment effects which are referred to in repeated measurements analysis. However, what may be sources of variation in a one-off measurements may become traceable to several identifiable sources in repeated measurements e.g. the differences due to a particular plot, particular animal, and preceding climate conditions.

\footnotetext{
*Address correspondence to this author at the AgResearch, P.O. Box 60,
} Lincoln, New Zealand; E-mail: scottd_hc@xtra.co.nz
Two of the commonly largest source of variation in grazing trial data is the difference between animals in performance and of possible variation in gut-fill on a particular weighing occasion. It has been noted that $12-23 \%$ of sheep or cattle bodyweight may be gut-fill, and that sheep may loose $5-6 \%$ of their weight in a $12 \mathrm{hr}$ fasting period [1,2]. Again it will be contended that while these are large sources of variability in one-period trials, that they become potentially quantifiable sources in repeat measurement trials.

One of the difficulties of stocking rate trials are differences in number of animals in different treatments leading to unbalanced design in some aspects of the analysis. One method of overcoming this is to vary the plot size so that the same numbers of animals are common to the different treatments.

The trial described was established with two complimentary objectives. The first was to establish by plant species selection, the degree of convergence in pasture composition from three alternative methods of sowing multispecies mixtures, and how the resulting pasture composition might be influenced by different grazing animal type and grazing pressure. Those results are described in [3].

The second objective, making use of the repeated grazing and duration necessary for the first, was to determine the animal weight gain responses in the same situation, but more particularly attempting to quantify the various factors besides main treatments which effect animal weight responses in those types of trials i.e. variation between animals, gut-fill and weather influences on particular weighing occasions, pasture type, plots, and climate growth conditions. Thus while it had an element of hypothesis testing (pasture sowing method and animal type), it was more an attempt to model 
animal weight changes under the various combination of conditions. This was attempted for conditions approaching marginality for such type of trials i.e. small numbers of near mature animals of different types, relatively small plots and not anticipated large differences due to pasture type.

There are two aspects of applied statistics - the computational methods leading to various descriptive parameters which are data neutral, and then any associated assumptions about any random nature and distribution of the variables leading to probabilistic estimates of the reliability or significance of those parameters. The trial used many of methods of the first stage to determine or adjust for the various effects but only made some comments on their possible application to the second stage.

\section{MATERIALS AND METHODS}

The trial was at AgResearch, Lincoln (New Zealand). The 1.0 ha trial of split-plot design of 24 fenced plots contrasted animal performance under treatments of 2 animal grazing types (sheep or goats) at 2 stocking rates (high or low, in ratio $3: 2$, by varying the fenced plot size), on a common mixed species pasture established by 3 contrasting methods, and with 2 spatial replications of all treatments.

The main plots were pasture sowing mixtures of 18 species established by 3 different methods drilled into fully cultivated high fertility developed pasture land on a Templeton soil. The sowing methods were: 1) a general mixture of all species; 2) each species or cultivar in a single coulter, with legumes and grasses in alternate coulters, and also cross drilled in the same manner, to give all species combinations; and 3) species divided into 18 random 4coulter mixtures with each species or cultivar present in a third of the mixtures. The four sub-plot treatments were the animal types by stocking rate treatments. The fenced plots were $38 \times 8.8 \mathrm{~m}$ (high stocking rate) or $38 \times 13.2 \mathrm{~m}$ (low stocking rate). The trial was sown in September 1988, first stocked in January 1989, and continuously stocked, except for two periods in July and mid August 1990, until May 1991.

The animals were 2-tooth wether sheep or goats, with each animal uniquely identified. Plots were grazed sequentially in groups of four for each animal type by stocking rate combination. The nominal duration on each set of plots was determined from pasture management considerations and usually 7 days (range 4-10 days depending on feed-on-offer). Most of the same animals made a total of 17 rotations through all plots during the trial period. During the initial 3 rotations, the sequence of movements through the plots was re-randomised. However, as that caused problems of differential pasture regrowth of plots, a fixed sequence was followed for later rotations. Because of the difference in size of animal types, the total stocking rates were approximately equalised between animal type in terms of total metabolic body weight per plot for reasonable utilization of the feedon-offer and potential pasture growth within the nominal week grazing period. Thus depending on the season, the number of animals varied from 4-8 sheep or 8-12 goats per plot, though maintaining equal number for the high and low stocking rates, and usually a constant number for each rotation through the 24 plots. While the variation in animal numbers between rotations had an element of a 'put-andtake' design, the responses of all animals were measured and used. The animals within each type were re-randomised between the two stocking rate treatments at each change. At the end of each grazing, animals were fasted overnight in a common paddock before weighing.

Prior to grazing the total feed-on-offer in each plot was determined from 70 capacitance probe measurements per plot. The residual feed-on-offer was similarly determined as animals were removed from plots. The capacitance probe measurements were converted to dry matter yields from calibration curves determined seasonally from cut samples. From these the feed-on-offer, residual, feed used, and in combination with total weight of animals per plot - the retrospective feed allowance was determined.

The vegetation composition of plots prior to grazing was determined by visually ranking the 12 most abundant species in each plot in order of their estimated contribution to shoot mass, and also the ratio of the relative contribution of two them to enable estimation of percentage contribution of all species [4]. The percentage contribution of the 7 most common species was used in the analyses. Pasture data was incomplete for three of the rotations.

The mean climate conditions for each period of air and soil temperatures, wind, solar radiation and Penman evaporation were obtained from the daily climate records from the meteorological station a kilometre away.

\section{SOURCES OF ADJUSTMENT AND ANALYSES}

\section{Weighing Day Gut-Fill}

The adjustments and analyses were done in a series of stages relating to the different sources of potential effects. The first was to consider the variability in animal weights relating to particular weighing occasions due to variation in animal gut-fill. While in practice this is minimized by fasting animals for a common period prior to weighing there is only limited experience on what variation there may be due to differences of a few hours or other features. There were some occasions in the present study when weights seemed universally above or below expectation based on the subsequently obtained longer-term weighing records.

The effect was quantified by indirect means. This was done by establishing the mean change in weight profile for the collective animal group across the whole trial period and using that to proportionally adjust individual animal weights for each particular weighing occasion. Animals were changed between treatments following weighing. Because of the experimental design of variable plot size with the same number of animals and including the same main treatments of animal type and stocking rate, it was reasonable to assume that the mean weight across all animals was only changing slowly and approximately linearly. The approach used was to assume the mean weight on each date in turn was missing and using linear regression of adjacent dates to estimate its value, and then determine the proportion of actual to estimate. It was assumed that this proportionality factor was principally related to variation in gut-fill and similar for all animals on each particular weighing occasion. This would have to be regarded as a generality and an approximation. 
Because of some addition or removal of animals, the particular aspects at the beginning or end of animal weight sequences, or periods when the plots were not being grazed, the procedure for estimating the moving average means weights varied. Within the main weekly sequence of dates the estimated means were based on actual weights on the two dates previous and the two dates following the particular weighing date. For weighing at one time interval from the end of the sequence the estimate was based on one before and the two after (or vise versa). For weightings at the beginning or end of sequences the estimates were based on the previously determined estimated means for the two adjacent dates. Proportionality estimates were restricted to the animals that were common to each of the relevant weighing occasions considered, though subsequently applied to all animals.

A further effect relating to particular weighing occasions is, with the fasting of animals in a common open paddock, of possible influence on observed weights from temperature, rain or dew deposition on surface wool or hair. That aspect was looked for as covariates in a quadratic response function between initial estimates of the date specific proportionality factor, and rainfall and grass minimum temperature in the day prior to weighing. The derived fitted proportionality factors were used to adjusted the observed weights of all animals. The alternate methods of moving average and weather effects on estimating the proportionality factors were iterated through five cycles before progressing to the growth rate estimation stage.

\section{Growth Rate Determination}

The second adjustment was the derivation of individual animal growth-rate parameters from their time/weight profile data, with a number of alternative expressions for animal performance.

The growth rate data was expressed as either the individual per animal performance, or as the collective for each plot and time period as the performance per area or hectare. For each animal and each time interval, growth rates were expressed as in either $\mathrm{g}$ day $^{-1}$ relative to measured weights, or as $\mathrm{g}$ day ${ }^{-1}$ relative to metabolic weight (measured weights**0.73), or as $\%$ day $^{-1}$ relative to their mean weight for the time interval. The three measures were hopefully successively more independent of animal type. The three parameters were appropriately summed or meaned for all animals on a plot to give corresponding per hectare performance. The covariates associated with each individual animal growth measurements were the individual animal identification, its mean weight for that period, and associated total animal weight ha ${ }^{-1}$.

\section{Between Animal Variability}

The third adjustment was to consider the between animal variability as a characteristic of individual animals rather than possible random or treatment effects. The individual animal effect could have two components - a general difference in performance relative to some group average, and a variation related to the general growing conditions. This is similar to the situation in plant evaluation studies of needing to quantify the difference between species or acces- sions across a range of environments and the corresponding technique of genotype/environment analysis seemed appropriate $[5,6]$. By analogy, that approach assumes a general scale for animal growing conditions can be defined by the mean performance of each group of animals, in each plot, in each period. Following that, the linear regression of the individual animal performance against the mean animal performance gives two coefficients, one an intercept describing the general difference for the individual animal, and secondly the gradient of how the performance of the individual animal changes relative to the average as general growing conditions change. While in the plant evaluation work the technique is used to highlight the difference between accessions, in the present context it was used to reduce the between animal variability by adjusting the individual animal response back to an average animal response, before determining treatment effects.

\section{Analysis}

The subsequent analyses were done at a number of levels.

(a) Variation in the base raw animal weights using analysis of variances computation as related to both weighing occasions and individual animal differences, or in relation to qualitative nominal treatment variables and weighing occasions.

(b) Similarly for the three growth rate parameters as either per animal or per hectare performance in relation to the main treatments and their interactions, treating those as qualitative nominal variables.

(c) Response functions using stepwise regression of the three growth rate parameters as either per animal or per hectare performance in relation to quantitative continuous independent covariable groups relating to measured aspects of the treatments. The covariable variables were for pasture yield, pasture composition, climate conditions and total grazing pressure during each period, and plot location. Treatments were also included as dummy continuous variables.

(d) The above analyses repeated to determine their stability under the likelihood that there was also measurement variability within the independent variables. This was done by introducing a reasonable random element into the values of the independent variables and seeing how they altered the analyses over a thousand iterations for each analysis, with the best one presented.

The decision in determining probabilistic predictions from most statistical analyses are whether particular treatments or effects are random or fixed effects, and accordingly which are the appropriate mean squares and comparisons for probabilistic inference. Those decisions are generally not inherent in the data and have to be determined from external contexts and considerations.

We would argue that in many grazing trials, like the present, those effects are fixed effects rather than random, with fixed effect models more appropriate than random effect models. In the present study sheep were sheep and goats were goats, selected for possible difference in grazing 
habit, and not a random two species sample of some infinite population of grazing-animal types. Also, while the animals used were intended as being "representative" or "random" for their selection going into the trial, once within the trial, they become fixed effects by individual identification. In the present study, with the identification and adjustment for individual animal effects, it could be debated whether they remained fixed effects, or following individual adjustment become more part of a random sample.

Two of the three pasture sowing methods were extremes; and selection of the two stocking-rates more akin to the construction of a spaced calibration curve than a random sample of all possible stocking-rates.
The results are given in terms of percentage variance accounted for in the response function regression approach and the corresponding fixed effect analysis of variance approach.

The particular feature of determining animal growth rates in repeat measurement trials is that what are final weights and time measurements for the end of one period also become the starting weights and time measurements for the next period. Thus variability in observation time and weight on one weighing occasion can influence two adjacent growth rate estimates. From one perspective that may lead to compensation in repeated measurements with the results being conservative, while from another perspective it may

Table 1. Animal Growth Parameters. Units, Means, Standard Deviations and Explanatory Variables, Together with Standard Deviation Used and Regression Coefficient in Simulation Stage

\begin{tabular}{|c|c|c|c|c|c|}
\hline Variable & Units & Mean & STD & Rand.std & Regress $\left(\times 1^{-3}\right)$ \\
\hline \multicolumn{6}{|l|}{ Growth rate } \\
\hline \multirow[t]{3}{*}{ - per animal } & $\mathrm{g} \mathrm{d}^{-1}$ & 7.72 & 367 & & \\
\hline & $\mathrm{gm} \mathrm{d}^{-1}$ & 2.03 & 101 & & \\
\hline & $\% \mathrm{~d}^{-1}$ & 0.018 & 1.06 & & \\
\hline \multirow[t]{2}{*}{ - per hectare } & $\mathrm{kg} \mathrm{d}^{-1}$ & 0.631 & 52.4 & & \\
\hline & $\% \mathrm{~d}^{-1}$ & 0.037 & 0.82 & & \\
\hline Animal weight & $\mathrm{kg}$ & 33.2 & 12.3 & 0.02 & \\
\hline Weighing date & $\mathrm{d}$ & na & 237 & 0.01 & \\
\hline Gut-fill proportion & & 1.0 & 0.032 & 0.005 & \\
\hline Animals per plot & $\mathrm{tha}^{-1}$ & 5.68 & 2.00 & & \\
\hline D. glomerata & $\%$ & 34.2 & 17.9 & & \\
\hline H. lanatus & “ & 21.2 & 14.0 & & \\
\hline L. hybridum & “ & 13.6 & 12.3 & 0.4 & -7 \\
\hline B. willdenowii & “ & 8.8 & 9.9 & 0.4 & -7 \\
\hline T. pratense & “ & 6.4 & 8.8 & & \\
\hline T. repens & “ & 3.5 & 4.0 & 0.4 & -9 \\
\hline F. arundenacea & “ & 4.8 & 5.8 & 0.4 & 5 \\
\hline \multicolumn{6}{|l|}{ Climate-weighing day } \\
\hline Rain & $\mathrm{mm}$ & 1.3 & 3.7 & 0.3 & \\
\hline Solar & & 14.4 & 7.3 & 0.2 & 30 \\
\hline \multicolumn{6}{|l|}{ Treatment } \\
\hline Rotation & $1-17$ & 8.8 & 5.0 & 0.01 & -237 \\
\hline Pasture & $0,1,2$ & 1.0 & 0.82 & 0.01 & -53 \\
\hline \multicolumn{6}{|l|}{ Location } \\
\hline $\mathrm{x}, \mathrm{y}$ & $\mathrm{m}$ & & & 0.3 & Fig. (1E) \\
\hline
\end{tabular}


obscure small but real effects. In the simulation phase of the study it was considered that the variation in weighing due to scale calibration, minor animal effects or recording on a particular occasion could be of the order of $\pm 0.2 \mathrm{~kg}$. The larger date effects relating to the likes of gut-fill were treated as a separate effect. In the present study, with weightings at nominally weekly intervals, the possible variation in time-ofday when only recorded as date was worth consideration (a few hours difference in weighing on a particular day could represent a c5\% difference in a weekly time interval). In the simulation stage this was initially estimated as of the order of \pm 0.01 day. In that stage the estimates of the both the weight and time variability's were applied prior to estimation of growth-rate measurements.

In the response function stage the nominal treatment variables were replaced with continuous covariables relating to pasture yield, grazing pressure, and pasture composition. Covariables were introduced to indicate any residual treatment effects as continuous variables (1-17 for rotation; 1, 0 for sheep versus goats; 0, 1 for low or high grazing pressure; $0,1,2$ for gradients of pasture mixtures; and 0,1 for the mixed pasture type versus the other two).

\section{Time and Other Variability}

Another aspect considered was the possibility of particular plot effects related to their geographic position. In its broadest form this was accommodated by designation of a spatial replication, though in the present work there was no known prior information why any environmental gradient should correspond with plot layouts. By adding covariates for the co-ordinates of plot centres, indices for each plot were estimated from a quadratic response relating to its location within the whole trial area.

Another consideration in repeat measurement trials is how to cope with adverse events, like instrument breakdown or occasional animal deaths. Where animals cannot be weighed, then their lack of measurement effects two determinations of growth rates, and both have to be treated as missing values. While their exclusion may have only limited effect in per animal analysis, it potentially has a larger effect on per hectare analysis, and a reasonable approach is to incorporate as a missing value the mean response of other animals in the same group.

\section{RESULTS}

\section{Data Sets}

The base data set from 105 weighing occasions gave 2583 observations for 94 interval periods of individual animal performances, of which 34 of 46 animals made up $95 \%$ of the observations. Animal data was missing for the 11 th rotation and for different groups of plots for part each of the 12 th and 17 th rotation. The corresponding condensed data set for plot per hectare performance contained 376 observations.

The mean and standard deviation of the variables showed a wide range in values (Table 1). The difference in number of observations between the response function and variance analyses relates to an incomplete set of covariate measurements.

The three data sets separating out the variables were for per area unadjusted, adjusted and random search analyses and results were those derived from the corresponding per animal data sets. The variance analyses for the random search phase were for the data sets which had given the best fit in the regression stage. The variance analyses for the random search time/weight animal profiles were from the data set which had given the best random search fit for the percent per day per animal data set.

\section{Weighing Occasion and Gut-Fill}

The mean weight of goats increased by only about a third over the two years of the trial while that of sheep nearly doubled (Fig. 1A). Both groups showed some loss during the first winter period and marked mean loss during the second autumn/winter period, particularly in the sheep. As a result there was a corresponding wide range in the calculated growth rate parameters. An initial variance analysis of the weights showed that $88 \%$ of the variation was accountable for from individual animal differences and weighing occasions, and alternatively only $49 \%$ related to treatments (Table 2).

The variability in the initial estimates of the proportionality factor between observed mean weight on each occasion, and that estimated from adjacent dates had a standard deviation of $3.21 \%$. Following the iteration procedure on the unadjusted data it was $3.05 \%$, with the suggested interpretation here, that that is an estimate of the variability in mean gut-fill relative to an average for each particular weighing occasion. The remaining $0.16 \%$ is attributed, by regression, to differences in grass minimum temperatures ( $=$ possible dew deposition) and rain in the over-night period during fasting and its effect on weight through surface wool or hair moisture content.

\section{Animal Variability}

There was wide and consistent differences between animals in their growth rates calculated from their individual time/weight profiles and unrelated to the particular treatments they were on. This is presented for 34 of the 46 animals with the most records expressed as their mean growth rate (as $\%$ day $^{-1}$ on horizontal axis) versus the rate at which that growth rate changed as general growing conditions improved (as $\left.\% \%^{-1} \mathrm{~d}\right)$, (Fig. 1B). The shaded two standard error bounds show there were differences between animals in both attributes.

There was no discernable difference in pattern between sheep and goats when growth rates were expressed on a percentage basis. There were no discernable trends in relationship between mean growth rates and the rate at which that might vary with general pasture growing conditions.

However, as the next sections show, adjustment for these individual animal effects increased the proportion explainable by 3.1-3.8 \% in variance analyses using qualitative treatment variables, and $2.7-3.7 \%$ in response function 
A. Time/weight

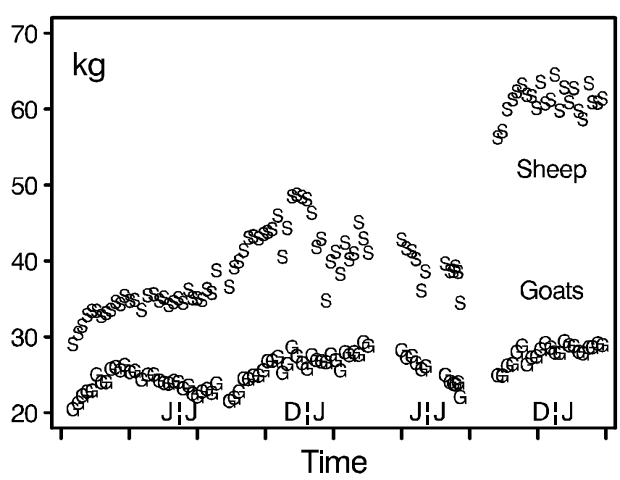

C. Location
B. Animal variability

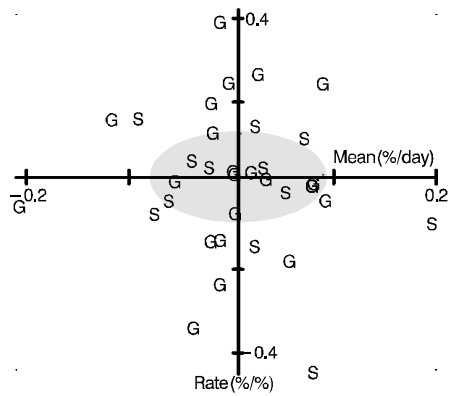

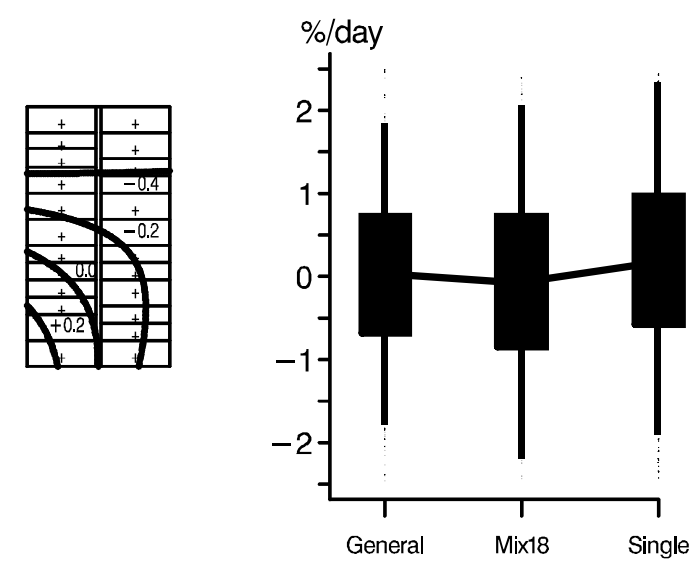

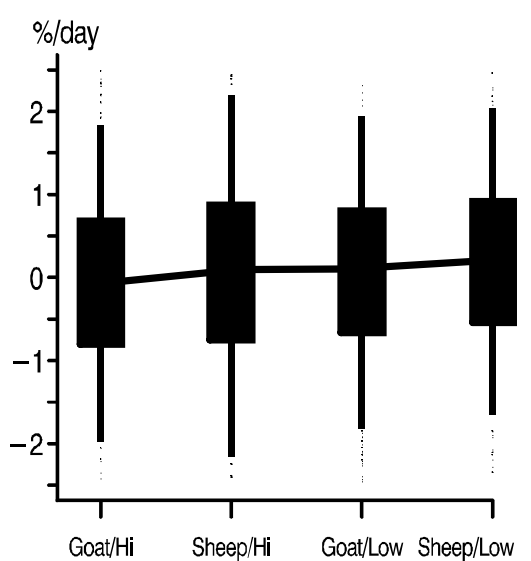

Fig. (1). Animal growth rate responses (\% weight gain $\left.\mathrm{d}^{-1}\right)$ of sheep and goats on three pasture mixtures. A. Changes in mean animal weight over trial period. B. Variability in individual animals as mean growth rate over trial period (horizontal axis) versus rate of change of growth rate with mean growing conditions (vertical axis). C. Influence of plot location on animal growth rates. D. Distribution of animal growth rates across three pasture types (box $25-75 \%$ percentile range, lines $1.5-98.5 \%$ percentile range). E. Distribution of animal growth rates in relation to animal type and grazing pressure.

analyses relative to measured climate, pasture, plot and collective animal covariates.

\section{Comparison of Variance and Response Function Analyses}

For the per animal growth rate parameters determined from the unadjusted raw data about half of the variance was explainable when treated as split-plot variance analysis of qualitative treatment variables and their interactions, but only $10-13 \%$ when treated as regression of continuous covariables (Table 2). In both, the explainable proportion was greatest when growth rates of both group of animals was expressed as $\% \mathrm{~d}^{-1}$ of mean weight of each animal, rather than as $\mathrm{g} \mathrm{d}^{-1}$ or $\mathrm{g}$ metabolic $\mathrm{d}^{-1}$, though the differences were small.

The explainable proportion increased when the same data sets were condensed into per area growth rate parameters and analysed.

In both forms of per animal analyses there was a reduction in the residual standard error of estimate when the base data was adjusted for the weighing occasion/gut-fill propor- tionality and for the individual animal effect. The trend was for the reduction to be the inverse of that for the previous growth rate parameter trend. We can offer no explanation for the difference in effect of these adjustments when the data was condensed into per area growth parameters, and the difference in the variance versus response function analyses.

In all the variance analyses some of the treatment effects would be significant in relation to the residual mean square if a fully fixed effect model was assumed, but no main effects would be significant compared to the appropriate interaction factors if a partial random effect model was assumed. Many of the groups and individual covariates would be significant relative to the residual standard error in the regression approach.

\section{Random Search Simulation}

Allowance for some variation in the recorded time intervals, animal weights and independent covariate values did show potentiality for an increase in the explainable variability. However, early simulations showed that the allowable variation could only be small. While the change in the value 
Table 2. Sources of Variation (\%) in Animal Growth Rates

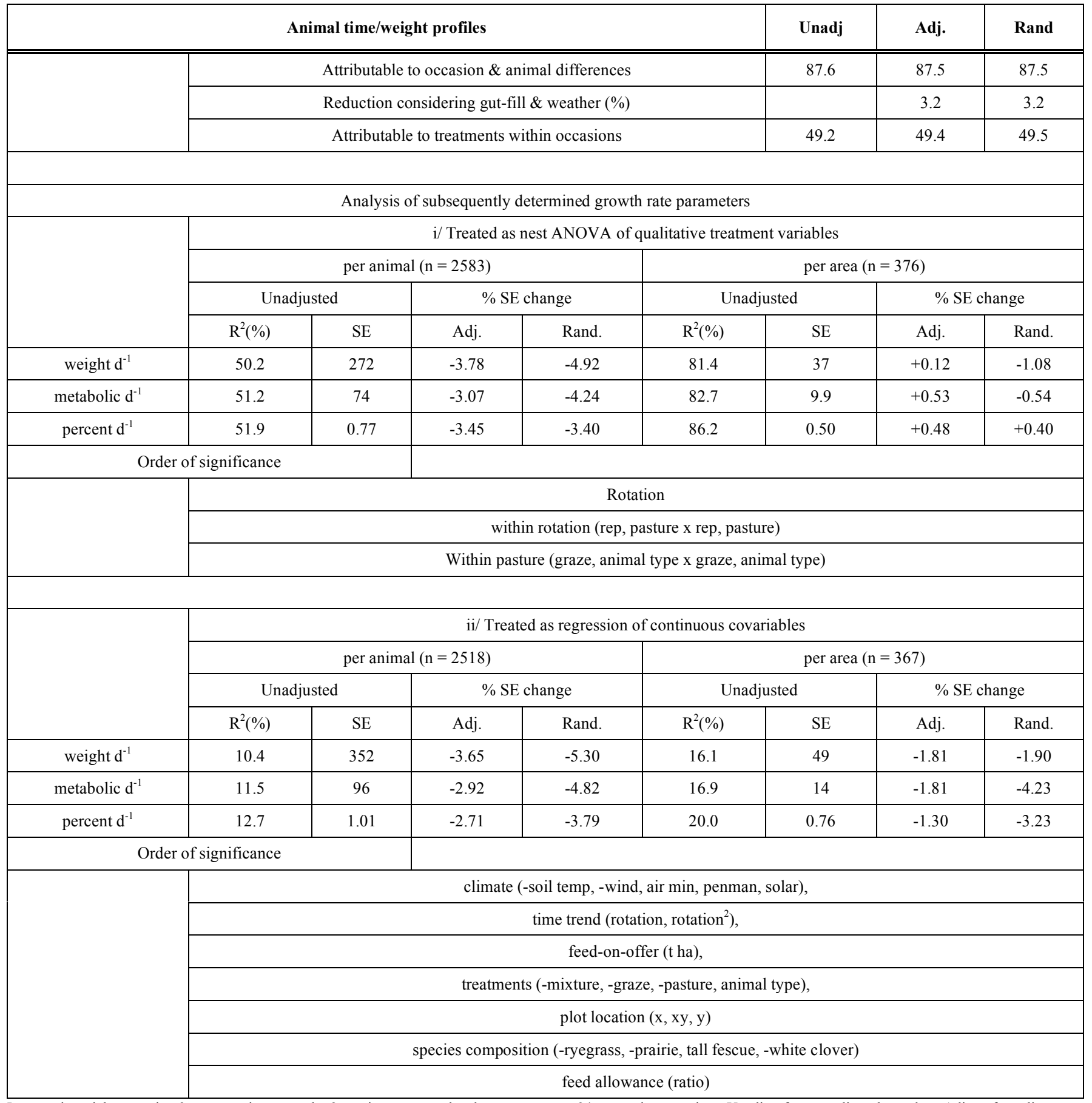

In a grazing trial comparing 3 pasture mixtures under 2 grazing pressures by sheep or goats on 94 successive occasions. Unadj. $=$ from unadjusted raw data. Adj. $=$ after adjustmen for gut-fill and for between individual animal variability. Rand. = after possible random variation in independent variables.

of a particular variable and observation within a likely range might be reasonable, the simultaneous random application to all variables and observations generally resulted in a greatly increased total variance, and the decreasing likelihood of finding a better fit within a reasonable number of simulations. The variables used, and their latitude in values as standard deviations of a normal distribution, are given in Table 2 .

In the best of a thousand iterations, there was a further small reduction in the explainable proportion of $0-1.2 \%$ and
$1.1-1.9 \%$ respectively for the variance or response function approach.

\section{Treatment and Covariate Effects}

The animal growth rates were lower on the 18 random species pasture mixture than on the general species mixture or the single species per coulter mixture (Fig. 1C). There was no reasonable biological explanation for this, though the two missing sets of data (from instrument break-down), and 
three of five other groups of somewhat doubtful data all deriving from those sets of plots.

There were the expected higher growth rates from the lower stocking rates (Fig. 1D). The growth rates of goats were generally lower than that of sheep by all three growth parameters and probably related to their greater maturity (Fig. 1A).

Though the proportion of variation accounted for was lower for the response function regression approach than the variance approach, because of the wider range and more explicit definition of covariates, they gave a more functional interpretation of influences. Most of the responses were sufficiently described by linear effects, with their magnitude given for their regression coefficient in per animal $\% \mathrm{~d}^{-1}$ base data adjusted analysis (Table 2).

The principal effects on animal growth rates were mean climate conditions during each grazing period, with a negative trend with soil temperature and wind, positive with air minimum temperature, Penman evaporation and solar energy, and no effect relative to other alternative air or soil temperatures or vapour pressure. These would have to be interpreted as probably effects on animals per se, as pasture and time effects were better described by other covariates.

There was a large residual time trend (rotation) probably relating to seasonal trends not adequately accommodated by temperature trends, and the increasing maturity of the sheep.

The feed-on-offer at the start of the grazing period was the best of the predictors from pasture covariates, followed by the feed allowance ratio. Three of the most common species in all pasture mixtures (Dactylis glomerata, Holcus lanatus, Trifolium pratense ([3]- Fig. 1)) were not discriminating variables in relation to any of the animal response parameters, with that role relating to some of the minor species (Lolium hybridum Hausskn, Bromus willdenowii Kunth, Festuca arundenacea Schreb, and Trifolium repens L.) and in a negative rather than positive effect.

There were residual treatment effects, the lower growth rates on the mixture pasture compared to the other two pasture types, the expected decrease with increasing stocking rate, and a small positive trend with the gradient from general mixture to single species row treatment.

There was a plot location effect with higher animal growth rates from plots in the lower left sector of the trial area (Fig. 1E). The mean weight of animals, or the total weight of animals on a plot, did not feature in any of the analyses.

\section{Adverse Events}

There were three adverse events during the course of the trial with two involving causalities during over-night fasting. In November (southern hemisphere) 1989 the goats were caught in irrigation spray during a cold storm with four deaths and five of the remaining showing unusually low liveweights. A cold storm in February 1990 gave one sheep casualty with four of the remaining sheep with unusually low live-weights. In trial management these were replaced with equivalent weight animals. In the random search analysis a dummy variable relating to these two groups of animals was introduced and included as a covariate in the simulation stage. Four occasions of apparently low sheep mean weights can be noted in Fig. (1A).

The other adverse effect was the rapidly reducing feed availability in the July-August period of the second year which was not adequately adjusted for in reduction of stocking rates. In management this was partly adjusted for by reduction in the days on the plots. However, the subsequently calculated low or negative daily growth rates were retained within the data set.

\section{DISCUSSION}

The proportion of variation accounted for by different animal growth-rate parameters in the present study from the various sources of potential variation was low. The estimates of the various effects had low reliability and only the high number of observations would have given significance in a probabilistic sense. The variance components were assessed using a fixed effect model, both because we believe that those better represent the nature of the variables and to allow comparison between the variance and regression model approach. If some of the variables are considered random, then the variance components would probably be better assessed by a mixed model repeat measurement design, though in this trial complex, rather than as a split-plot design as given. However, the study has indicated that it is possible to consider and partially quantify effects in repeated measurement trials which are sources of uncertainty and included in the error term in one-off one-period trials. The present work should be viewed in terms of methodology suggested rather than for the particular biological results obtained.

Another incentive for the present work was the desire to replace the qualitative nominal treatment variables (pasture, stocking-rate etc.) used at the design stage with measured functional variables (pasture yields, species composition, total $\mathrm{kg} \mathrm{ha}^{-1}$ etc.) at the analysis stage, and change towards a response function approach. Associated with that is the growing consensus that in the design of grazing trials with limited resources, that further stocking rate or other treatments of variables having direct functional interpretation, are more valuable, that spatial replications per se $[7,8]$. The response function approach is also considered more useful for subsequent incorporation into modelling or simulation studies [9].

Any such study is heavily dependent on the accuracy in the base data. The present trial, with often very small differences in animal weights at weekly intervals, emphasised the desirable practice of weighing each animal independently at least twice on each weighing occasion, the frequent taring of scales or insertion of dummy 'animals' or objects of known standard weights, and retaining all records, particularly the original field records, so that any doubtful records in the final data set can be fully traced. That was not sufficiently done in the present work.

Animal growth rates can be expressed in a number of ways, on per animal or per area rate using different parameters. In the present trial a higher proportion of the variation was explainable considering per area rates with the difference between animal types best accounted for using percentage per day weight gains. 
The trial has also suggested an indirect method of adjusting for the common uncertainty in live-weight gains in grazing trials of the effect of gut-fill on particular weighing occasions. The approach does depend on repeat measurements to get reasonable estimates of moving averages and the assumption that mean departures do relate to gut-fill rather than treatment or other affects. It also has to assume, at least as a first approximation, that the adjustment is a mean factor common for all animals on a particular weighing occasion. It is likely to work best, as in this trial, where animals were re-allocated between treatments in each interval. In the present trial the approach did make a useful reduction in the between animal variability for subsequent analyses of treatment or covariate effects.

The application of the genotype/environment approach was to make adjustment for individual animal variability in trials using few animals where there were repeat measurements. In one sense it was an attempt to calibrate individual animal's responses relative to some mean response so that they could correctly measure the responses in whatever treatment combination they were placed.

In those adjustments there were no discernable trends in relationship between mean growth rates and the rate at which that might change with general growing conditions (Fig. 1B) as there often is in the case in plant studies of genotype /environment form of analysis. However, the approach did make a useful reduction in the between animal variability for subsequent analyses of treatment and other effects.

While the genotype /environment form of analysis was used in the present context to reduce the between-animal variability prior to analysis for treatment effects, in a different context it could be used to select animals of superior growth-rates, and either more superior under better growing conditions (high values of rate of change) or more consistent across a range of growing conditions (low values of rate of change), as in plant evaluation studies.

There has been much discussion on the most appropriate model structure for the analysis of grazing trial data with their often repeated measurements on the same animals, and the low number of degrees of freedom for the error term when there are a low number of animals or treatments [1014]. The present work has probably not taken cognisance of all the issues raised in those discussions, with its emphasis on the response function approach. The present study had a large element of repeat design, but with the repeat at six time intervals.

What does not seem to have been adequately considered is the change in the most appropriate statistical model structure that may occur in the transformation from weight/time profiles to rate of growth measures for the successive intervals. In one sense it could be argued that the transformation to growth rate increments uncouples the observations from the repeated measurement constraint. In another sense, as referred to in the present work, is the effect of uncertainty or error in each weight measurement, and its influence on to two adjacent growth rate measurements. The random search simulation in the present study was an attempt to determine the likely magnitude of those effects. They illustrated that there could be small increase in the explainable variability.
In trials where the animals are retained on the same treatments Gill [12] has suggested fitting polynomial regressions to each animal weight profile before analysis. It would seem that differentiation of each of the fitted regression would enable direct estimate of the growth rates for the different time intervals. Gill [12] was suggesting polynomial regressions to the level considered appropriate. However, for trials where there was a high number of repeat measurements, such as might have been in the present trial if the individual animals had remained on the same treatments, and responses were being more influenced by outside factors such as season, then the polynomial model could become unduly complex, and if the differentiation option used were determined by the whole sequence of measurements rather than those in proximity to particular periods. Initial spline fitting would seem more appropriate to confine estimates to each section of the total weight profile.

The random search simulation stage was an attempt to gauge the effect of reasonable measurement uncertainty on the results. There were two aspects, the effect of reasonable uncertainty of measurement of time intervals and weights on subsequently calculated animal growth rates, and the unlikely usual statistical assumption that independent variables are measured without error. As judged by the best of a thousand simulations, allowable reasonable uncertainty could further slightly increase explainable variability.

In summary the suggestion is that it is possible to identify and quantify some of the effects in animal growth rate studies in few-animal short-period repeat measurement trials which are not available in one-of trials. This through

- replication or checking of initial animal weight measurements.

- adjustment for weighing-day/ gut-fill weights via moving averages of mean weights.

- $\quad$ calibration and adjustment for individual animal responses by a genotype/environment approach.

- use of percent weight gains rather than absolute weight gains.

- $\quad$ consideration of variability in independent variables as well as dependent variables.

\section{REFERENCES}

[1] Hughes JG. Short-term variation in animal liveweight and reduction of its effect on weighing. Anim Breed Abst 1976; 44: 111-18.

[2] Stuedemann JA, Matches AG. Measurement of animal response in grazing research. In: Martin GC, Ed. Grazing research: Design, methodology, and analysis. Madison, Wisconsin, USA: Crop Science Society of America Inc. \& American Society of Agronomy Inc. 1989; 21-35.

[3] Scott D, Penell CGL. Agronomic methods: Evaluation by multiple species pasture mixtures. NZ J Agr Res 2006; 49: 191-200.

[4] Scott D. Description of vegetation interactions using visual ranking of species. NZ J Ecol 1989; 12: 77-88.

[5] Finlay KW, Wilkinson GN. The analysis of adaption in a plant breeding programme. Aust J Agr Res 1963; 14: 742-54.

[6] Scott D. Plant introduction trials: genotype-environmental analysis of plant introductions for the high country. NZ J Exp Agric 1985; 13: 117-27.

[7] Riewe ME. Use of relationship of stocking rate to gain of cattle in an experimental design for grazing trials. Agron J 1961; 53: 309-13 
[8] Bransby DI. Compromise in the design and conduct of grazing experiments. In: Martin GC, Ed. Grazing research: Design, methodology, and analysis. Madison, Wisconsin, USA: Crop Science Society of America Inc. \& American Society of Agronomy Inc. 1989; 53-67.

[9] Loewer OJ. Issues on modelling grazing systems. In: Martin GC, Ed. Grazing research: Design, methodology, and analysis. Madison, Wisconsin, USA: Crop Science Society of America Inc. \& American Society of Agronomy Inc. 1989; 127-36.

[10] Gill JL, Hafs HD. Analysis of repeated measurements of animals. J Anim Sci 1971; 33: 331-6.

[11] Allen OB, Burton JH, Holt JD. Analysis of repeated measurements from animal experiments using polynomial regression. J Anim Sci 1983; 57: 765-70.
[12] Gill JL. Repeated measurement: sensitive tests for experiments with few animals. J Anim Sci 1986; 63: 943-54.

[13] Drane JW. Compromise and statistical design for grazing experiments. In: Martin GC, Ed. Grazing research: Design, methodology, and analysis. Madison, Wisconsin, USA: Crop Science Society of America Inc. \& American Society of Agronomy Inc. 1989; 69-83.

[14] Giesbrecht FG. Experimental design and statistical inference: Generalized least squares and repeated measures over time. In: Martin GC, Ed. Grazing research: Design, methodology, and analysis. Madison, Wisconsin, USA: Crop Science Society of America Inc. \& American Society of Agronomy Inc. 1989; 85-96.

(C) David Scott; Licensee Bentham Open.

This is an open access article licensed under the terms of the Creative Commons Attribution Non-Commercial License (http://creativecommons.org/licenses/by$\mathrm{nc} / 3.0 /$ ), which permits unrestricted, non-commercial use, distribution and reproduction in any medium, provided the work is properly cited. 